\title{
Budd-Chiari syndrome with portal, splenic, and superior mesenteric vein thrombosis treated with TIPS: who dares wins
}

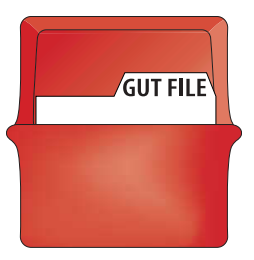

\section{PATIENT/HISTORY}

A 20 year old man presented with severe ascites and malnutrition due to BuddChiari syndrome (BCS) with portal vein $(\mathrm{PV})$, and splenic and mesenteric vein (SMV) thrombosis in the proximal $3 \mathrm{~cm}$. $\mathrm{He}$ had received regular and more frequent paracenteses of up to 17 litres each for eight months. He had a poor BCS prognostic index of $8.4^{1}$ (table 1). After referral, despite full anticoagulation and diuretic drugs, he deteriorated over three weeks. No thrombophilic disorder was found.

\section{DIAGNOSTIC PROCEDURES/ INTERVENTION}

He underwent transjugular intrahepatic portosystemic stent shunt (TIPS) under general anaesthesia. As all hepatic veins were completely occluded, a direct inferior vena cava (IVC) puncture was made. No patent major PV tributaries were demonstrated; two capsular punctures were embolised. Eventually, a small PV tributary was cannulated and a catheter advanced into the SMV. Because of extensive thrombosis, no PV pressure was recordable; right atrium (RA) 12 $\mathrm{mm} \mathrm{Hg}$, IVC $21 \mathrm{~mm} \mathrm{Hg}$. The parenchymal tract was dilated and two overlapping $12 \mathrm{~mm}$ Memotherm stents deployed. The SMV and PV thrombus was partially mechanically disrupted by an angiographic pigtail catheter. Anticoagulation was not performed due to the risk of developing intrahepatic haematoma. Two days later, further thrombus was again mechanically disrupted and one Memotherm stent was overlapped in the upper and one in the lower end of the TIPS tract. Good flow was re-established and pressures were $(\mathrm{mm}$ $\mathrm{Hg}$ ): PV 33, IVC 22, portosystemic gradient 11, and RA 6. Heparin was started. A widely patently TIPS shunt with excellent flow was present a week later with an RA pressure of $13 \mathrm{~mm} \mathrm{Hg}$ and PV $23 \mathrm{~mm} \mathrm{Hg}$. Warfarin was commenced.

\section{OUTCOME}

The patient improved progressively, only one paracentesis was needed, ascites resolved completely, and diuretics were stopped after 45 days. At 60 days he had improved dramatically, with no ascites, and body weight increasing by $12 \mathrm{~kg}$ (table 1).

\section{DISCUSSION}

BCS can be associated with splanchnic venous thrombosis in up to $20 \%$ of patients, and this association worsens prognosis. ${ }^{2}$ If the thrombus extends down the SMV, both radiological and surgical intervention, including liver transplantation, are contraindicated in many centres. In this setting, successful treatment with transcaval liver resection and hepatoatrial anastomosis (Senning's procedure) has been reported. ${ }^{3}$ However, this surgery requires sternotomy and laparotomy, and cannot be performed in severely ill patients such as ours who was severely malnourished and with worsening hepatic function. In contrast, TIPS has been reported in single BCS patients not responding to medical therapy and with portal vein thrombosis alone. ${ }^{4}$

Our patient shows that extensive splanchnic venous thrombosis in patients with BCS is not an absolute contraindication for TIPS and is worth attempting even when SMV is thrombosed. There is only one previous case report in which TIPS was used in this situation: TIPS was successful and the patient was doing well after 18 months.

In conclusion, we believe that TIPS should be attempted in patients with BCS even when splanchnic veins are extensively thrombosed. It can provide the only therapeutic avenue, improvement can be spectacular, and liver transplantation can be avoided

A Mancuso

Universita' di Palermo, Scuola di Specializzazion in Gastroenterologia ed Endoscopia Digestiva Reparto di Medicina, Ospedale V Cervello, Via Trabucco 180, 90144, Palermo, Italy

A Watkinson, J Tibballs, Department of Radiology, Royal Free Hospital, London, UK

D Patch, A K Burroughs, Liver Transplantation Unit and Hepatobiliary Medicine, Royal Free Hospital, London, UK, andrew.burroughs@talk21.com

\section{REFERENCES}

1 Valla D. Hepatic vein thrombosis (Budd-Chiari syndrome). Semin Liver Dis 2002;22:5-14.

2 Mahmoud A, Helmy A, Billingham L, et al. Poor prognosis and limited therapeutic options in patients with Budd-Chiari syndrome and portal venous system thrombosis. Eur J Gastro Hepatol 1997;9:485-9.

3 Sauvanet A, Panis Y, Valla D, et al. Budd-Chiari syndrome with extensive portal thrombosis: treatment with Senning's procedure. Hepatogastoenerology procedure. Hepa

4 Perello A, Garcia-Pagan JC, Gilabert R, et al. TIPS is a useful long-term derivative therapy for patients with Budd-Chiari syndrome uncontrolled by medical therapy. Hepatology 2002;35: 132-9.

Hepatology 2002;35:132-9. 5 Pfammatter T, Benoit C, Cathomas
Budd-Chiari syndrome with splenomesenteric-portal thrombosis: treatment with extended TIPS. J Vasc Interv Radiol $2000 ; 11: 781-4$.

Table 1 Comparison of laboratory and clinical data before and two months after transjugular intrahepatic portosystemic stent shunt (TIPS)

\begin{tabular}{|c|c|c|}
\hline Parameter (normal values) & Before TIPS & After TIPS \\
\hline Weight (kg) (height $170 \mathrm{~cm}$ ) & 45 & 56 \\
\hline Haemoglobin g/l (1 1.5-15.5) & 8.2 & 11.6 \\
\hline White blood cells $\left(\times 10^{9} / 1\right)(3.2-11)$ & 12.2 & 5.5 \\
\hline Platelets $\left(\times 10^{9} / 1\right)(140-400)$ & 260 & 308 \\
\hline International normalised ratio (0.9-1.2) & $2.3^{*}$ & $2.3^{*}$ \\
\hline Alanine aminotransferase $(\mathrm{u} / \mathrm{l})(5-40)$ & 24 & 27 \\
\hline Aspartate aminotransferase $(\mathrm{u} / \mathrm{I})(5-40 \mathrm{O})$ & 46 & 37 \\
\hline Bilirubin $(\mu \mathrm{mol} / \mathrm{l})(5-17)$ & 66 & 51 \\
\hline Albumin $(g / I)(35-50)$ & 27 & 39 \\
\hline Creatinine ( $\mu \mathrm{mol} / \mathrm{l})(60-120)$ & 66 & 45 \\
\hline Ascites & Yes & No \\
\hline Encephalopathy & No & No \\
\hline Child-Pugh class & C & B \\
\hline Diuretics & Yes & No \\
\hline Budd-Chiari syndrome index $>5.4$ & Yes & No \\
\hline
\end{tabular}

